\title{
Prevalence of Vitamin D Deficiency in Obstructive Sleep Apnea Disorder in Dubai, UAE
}

\author{
Fatehrahman Shendi*, Bassam Mahboub, Hassan Alhariri and Annie Soans
}

Sleep Disorders Center, Department of Medicine, Rashid Hospital, Dubai Health Authority, Dubai United Arab Emirates

\begin{abstract}
Purpose: Sleep disorders have become epidemic throughout the worldwide, at the same time due to Multiple social changes, the same thing can be said for the vitamin D deficiency disorders, including the use of air conditioning and sunscreen, have produced a world-wide epidemic of vitamin D deficiency, then taking in consideration the proposed concept that since vitamin D plays an important role in the brainstem control of sleep, which is supported by the presence of vitamin $D$ receptors in same areas are considered to play a role in the initiation and maintenance of sleep, this relation may not be strong as causative association but presence of either may have worsening effect on the other.
\end{abstract}

Methods: The aim of our study was to investigate the prevalence of between serum 25-hydroxy [25 (OH)] vitamin $\mathrm{D}$ levels and severity of disease in patients with OSAS, among the cosmopolitan (multi-national) population of Dubai, United Arab Emirates. Two hundred and sixty nine (269) patients were included in the study, all who were referred to the sleep clinic at Rashid hospital - Dubai health Authority, during the period of three years from 2012 to 2014 . Serum 1-25 Di Hydroxy Chole Caciferaol (vitamin D), were evaluated.

Results: Among the 148 patients who had OSA; those who were vitamin D deficient represent $16.1 \%$ of the Mild OSA group, $7.3 \%$ of the moderate OSA group and $7.9 \%$ of the Severe OSA group, while at the same time those who were vitamin D insufficient represent $29 \%$ of the Mild OSA group, $39 \%$ of the moderate OSA group and $31.6 \%$ of the Severe OSA group.

Conclusions: When the severity of OSA increases, then vitamin deficiencies, becomes more pronounced(but not significant), Thus subjects with more severe OSA indices tended to present lower vitamin D levels; furthermore Vitamin D deficiency may play a role and/or worsen OSA's. We need to study more OSA adverse outcomes on glucose metabolism, in relation to vitamin D deficiency \& insufficiency, as may be considered for supplementation treatment.

Keywords: Obstructive sleep apnea; Snoring; Daytime sleepiness; Prevalence primary care; Berlin questionnaire; Dubai

Abbreviations: BMI: Body Mass Index; OSAS: Obstructive Sleep Apnea Syndrome; DHA: Dubai Health Authority; PHC: Primary Health Care; UAE: United Arab Emirates

\section{Introduction}

\section{Vitamin D facts}

Vitamin D is often referred to as the sunlight vitamin, because skin or dermal synthesis is the main manufactures and is the major natural source, Pre-vitamin D3 is synthesized non-enzymatically from 7-dehydrocholesterol during exposure to the ultraviolet (UV) rays in sunlight, then undergoes a temperature-dependent re-arrangement to form vitamin D3 (cholecalciferol). Vitamin D is also present in Very few and limited foods naturally, including dairy products, fatty fish like tuna and fortified breakfast cereals [1]. Thus It's often hard to meet daily requirement of vitamin $\mathrm{D}$ from food sources alone, thus may increase risk of a vitamin $\mathrm{D}$ deficiency if individuals receive limited exposure to sunlight. Dietary vitamin D is incorporated into micelles, absorbed by enterocytes, and then packaged into chylomicrons. Thus Disorders associated with fat malabsorption, such as celiac disease, Crohn disease, pancreatic insufficiency, cystic fibrosis, short gut syndrome, and cholestasis liver disease, are associated with low serum 25-hydroxyvitamin D (25[OH]D) levels.

Vitamin D is a hormone that interacts with intra-nuclear receptors to effect transcriptional changes in many cell types including those in gut, bone, breast, prostate, brain, skeletal muscle, and the immune system.

Vitamin D deficiency or Inadequacy of vitamin D is widely prevalent, may place individuals at a higher risk of bone weakness and leads to the classic diseases of bone demineralization problems, nonspecific pain and non-inflammatory skeletal myopathy, as well as to more recently recognized linked to a higher incidence of certain types of cancer, depression and obesity.

Furthermore Emerging lines of evidence suggest that low vitamin D levels increase the risk for autoimmune disease, chronic rhinitis, adeno-tonsillar hypertrophy, airway muscle myopathy, and/or chronic rhinitis, cardiovascular disease, and diabetes, all these conditions are mediated by altered immunomodulation, increased propensity to infection, and increased levels of inflammatory substances, including those that regulate sleep, such as tumor necrosis factor alpha (TNF- $\alpha$ ),

${ }^{*}$ Corresponding author: Fatehrahman Shendi, MD, Sleep Disorders Center Department of Medicine, Rashid Hospital, Dubai Health Authority P.O.box 4545, Dubai, United Arab Emirates, Email: fatehrahman@hotmail.com

Received March 09, 2016; Accepted May 10, 2016; Published May 13, 2016

Citation: Shendi F, Mahboub B, Alhariri H, Soans A (2016) Prevalence of Vitamin D Deficiency in Obstructive Sleep Apnea Disorder in Dubai, UAE. J Clin Respir Dis Care 2: 112. doi: $10.4172 / 2472-1247.1000112$

Copyright: (C) 2016 Shendi F, et al. This is an open-access article distributed under the terms of the Creative Commons Attribution License, which permits unrestricted use, distribution, and reproduction in any medium, provided the original author and source are credited. 
interleukin (IL)-1, and prostaglandin D2 (PD2) [2].

Vitamin D plays an important role in the brainstem control of sleep [3], which is supported by the presence of vitamin D receptors in the anterior and posterior hypothalamus, substantia nigra, midbrain central gray, raphe nuclei, and the nucleus reticularis pontis oralis and caudalis, which these same areas are considered to play a role in the initiation and maintenance of sleep, thus all of which may be key causes of the development of symptoms of daytime wake impairment with sleep disrupted disorders, and sleep apnea.

Many Multiple social changes, including the use of air conditioning and sunscreen, have produced a world-wide epidemic of vitamin D deficiency and study the relation between sleep and vitamin $\mathrm{D}$ is found to be mandatory, and require attention of the research community.

\section{Sleep Apnea Facts}

Obstructive sleep apnea/hypopnea syndrome (OSAHS) is a highly prevalent disorder with multiple comorbidities.

OSAHS is characterized by repetitive episodes of airflow reduction (hypopnoea) or cessation (apnoea) due to upper airway collapse during sleep [4]. Its major risk factor is obesity. However, its pathogenesis is complex and multifactorial. Reduced upper airway muscle tonus and/ or unstable neuromuscular output seem to be involved in this collapsus

A normal vitamin D status is necessary for normal muscle function and neuromuscular output. As obesity is associated with a high rate of hypo-vitaminosis D, it appears of interest to evaluate the Association between presence and severity of OSAS and vitamin D level. Some practitioners, such as neurologist Stasha Gominak, believe that vitamin D deficiency can cause sleep apnea and other sleep disorders. Vitamin D supplementation may help, although clinical research evaluating this claim is still ongoing $[5,6]$. The aim of our study was to investigate the prevalence of between serum 25-hydroxy [25 (OH)] vitamin D levels and severity of disease in patients with OSAS, among the cosmopolitan (multi-national) population of Dubai, United Arab Emirates.

\section{Methodology}

The study consists of 269 patients, represents the multi-national and cosmopolitan community of Dubai, United Arab Emirates (UAE), those who were referred to the sleep clinic in Rashid Hospital - Dubai Health Authority (DHA) for investigation of obstructive sleep apnea (OSA), were at the same time investigated for the serum level of vitamin D.

Patient with OSA were classified depending on Apnea HypoApnea index (AHI) score on the sleep study, according to the American Academy of Sleep medicine scoring and classification criteria and guidelines, in to, non OSA if AHI were below 5, mild if AHI were between 5-15, moderate if AHI were 15-30 and sever if AHI were above 30 . While serum level of vitamin $\mathrm{D}$ were classified, depending on serum level referring to American college of endocrinology criteria and guidelines, into, of normal value if above 30 , insufficient if below 30 , and deficient if below 10, Then percentage calculation were performed to estimate the relevance of OSA and Vitamin D deficiency or insufficiency and represented in table, further subcategories of patients performed and calculation done to explore the similar percentages, among local UAE nationals and expatriates UAE residents, and further comparisons.

\section{Results}

The study consists of 269 patients of which $45 \%$ are in the age group
40 to 60 years, $28 \%$ are older than 60 years and $27 \%$ are between 14 to 39 years, $53.2 \%$ are female and $46.8 \%$ are males, with $73 \%$ of the participating patients are local UAE Nationals and $27 \%$ are expatriate UAE residence patients (Table 1).

Out of the 59 patients with No OSA, 17.9\% are Vitamin D deficient and $26.8 \%$ are Vitamin D insufficient. On the other hand the remaining who had OSA; $11.8 \%$ of the Mild OSA group, $7.3 \%$ of the moderate OSA group and $7.9 \%$ of the Severe OSA group are Vitamin D deficient (Table 2).

At the same time $30 \%$ of the Mild OSA group, $39 \%$ of the moderate OSA group and $31.6 \%$ of the Severe OSA group are Vitamin D insufficient (Table 3).

When we looked at the 144 patients who had the vitamin D level checked, we found that; 23 patients had normal vitamin D levels, 84 patients had vitamin D insufficiency and 30 patients had vitamin D deficiency [7].

Out of the 30 patients with No OSA, $13.3 \%$ had normal vitamin D, $53.3 \%$ had vitamin D insufficiency and $33.4 \%$ had vitamin D deficiency. Of the 45 patients with mild apnea, $13.3 \%$ had normal vitamin D, $62.3 \%$ had vitamin D insufficiency and $24.4 \%$ had vitamin D deficiency. While among the 23 patients with moderate apnea, $17.5 \%$ had normal vitamin $\mathrm{D}, 69.5 \%$ had vitamin $\mathrm{D}$ insufficiency and $13 \%$ had vitamin $\mathrm{D}$ deficiency. In the 39 patients with severe sleep apnea we found $23 \%$ had normal vitamin $\mathrm{D}, 61.5 \%$ had vitamin $\mathrm{D}$ insufficiency and $15.5 \%$ had vitamin $\mathrm{D}$ deficiency in general. There was no statistically significant difference in serum 25(OH)D levels between OSAS and controls ( $\mathrm{p}>0.05)$ (Table 4).

Of these 144 patients who had the vitamin D level checked, 23 patients had normal vitamin D levels, out of them $17.4 \%$ with No OSA, $26 \%$ with mild osa, $17.4 \%$ with moderate osa and $39 \%$ with severe osa [8]. Of the 84 patients with vitamin D insufficiency, we found $19 \%$ with No OSA, $33.3 \%$ with mild OSA, $19 \%$ with moderate osa and $28.7 \%$ with severe osa, While among the 30 patients with vitamin $\mathrm{D}$ deficiency we found $33.4 \%$ with No OSA, $36.6 \%$ with mild osa, $10 \%$ with moderate osa and $20 \%$ with severe osa.

In this subgroup analysis of OSAS, we found that the patients with severe OSAS had significantly lower levels of $25(\mathrm{OH}) \mathrm{D}$ as compared with other groups $(\mathrm{p}=0.003)$.

Also, the number of patients with serum 25(OH)D deficiency $(<10$ $\mu \mathrm{g} / \mathrm{dl})$ were higher in OSAS group than in controls $(\mathrm{p}<0.05)$ (Table 5).

\section{Discussion}

Many clinical studies emphasized the direct relation between increased incidence of insulin resistance (IR) $[9,10]$, type two diabetes mellitus (type $2 \mathrm{DM}$ ) and OSA. Obesity is known to be an important risk factor for both OSA and abnormal glucose metabolism.

There is growing evidence suggesting that low-grade systemic inflammation may serve as the mechanistic link between OSA and metabolic disorders including IR. Vitamin D insufficiency has long been suspected as a risk factor for glucose intolerance and diabetes. The serum concentration of 25-hydroxyvitamin-D3 (25-OH-D), which also defines the nutritional status, was shown to be lower in obese and/or diabetic patients and in those who were at high risk for diabetes. Adipose tissue, which is a well-known source of inflammatory cytokines, may be responsible for the decreased bioavailability and enhanced metabolic clearance of vitamin D in obese subjects.

Many arguments can be presented in the recent few years regard 
Citation: Shendi F, Mahboub B, Alhariri H, Soans A (2016) Prevalence of Vitamin D Deficiency in Obstructive Sleep Apnea Disorder in Dubai, UAE. J Clin Respir Dis Care 2: 112. doi: 10.4172/ 2472-1247.1000112

Page 3 of 4

\begin{tabular}{|c|c|c|}
\hline Age groups (years) & Frequency & Percent \\
\hline 14 to 39 & 74 & 27.5 \\
\hline 40 to 60 & 120 & 44.6 \\
\hline Older than 60 & 75 & 27.9 \\
\hline Total & 269 & 100 \\
\hline Sex & Frequency & Percent \\
\hline Female & 143 & 53.2 \\
\hline Male & 126 & 46.8 \\
\hline Total & 269 & 100 \\
\hline Nationality & Frequency & Percent \\
\hline Local & 197 & 73.2 \\
\hline Non local & 72 & 26.8 \\
\hline Total & 269 & 100 \\
\hline BMI groups & Frequency & Percent \\
\hline$<20$ & 11 & 4.1 \\
\hline $20-30$ & 54 & 20.1 \\
\hline $30-40$ & 97 & 36.1 \\
\hline $40-50$ & 64 & 23.8 \\
\hline Above 50 & 39 & 14.5 \\
\hline Data not available & 4 & 1.5 \\
\hline Total & 269 & 100 \\
\hline
\end{tabular}

Table 1: The study showed $45 \%$ of the patients $\%$ are in the age group 40 to 60 years and only $20 \%$ of them had a normal BMI.

\begin{tabular}{|l|l|l|l|}
\hline OSA/No OSA & Frequency & Percent \\
\hline OSA & & 210 & 78 \\
\hline$\bullet \quad$ Mild & 93 & 34.5 \\
\hline$\quad$ Severe & 41 & 15.2 \\
\hline No OSA & 76 & 28.3 \\
\hline Total & 59 & 22 \\
\hline
\end{tabular}

Table 2: The study showed $78 \%$ of the patients have Obstructive Sleep Apnea ( $35 \%$ have mild OSA, $15 \%$ have moderate OSA and $28 \%$ have severe OSA), and $22 \%$ do not have OSA.

\begin{tabular}{|c|c|c|c|c|c|}
\hline \multirow{2}{*}{$\begin{array}{l}\text { OSA Severity (all } \\
\text { patients) }\end{array}$} & \multicolumn{4}{|c|}{ Vitamin D problem } & \multirow{2}{*}{ Total } \\
\hline & Not done & Normal & Vitamin D insufficiency & Vitamin D deficiency & \\
\hline No OSA & $29(48.2 \%)$ & $4(7.1 \%)$ & $16(26.8 \%)$ & $10(17.9 \%)$ & 59 \\
\hline Mild & $48(51.6 \%)$ & $6(6.4 \%)$ & $28(30.2 \%)$ & $11(11.8 \%)$ & 93 \\
\hline Moderate & $18(43.9 \%)$ & $4(9.8 \%)$ & $16(39.0 \%)$ & $3(7.3 \%)$ & 41 \\
\hline Severe & $37(48.7 \%)$ & $9(11.8 \%)$ & $24(31.6 \%)$ & $6(7.9 \%)$ & 76 \\
\hline Total & $132(49.1 \%)$ & $23(8.5 \%)$ & $84(31.2 \%)$ & $30(11.2 \%)$ & 269 \\
\hline
\end{tabular}

Table 3: The relationship between vitamin $\mathrm{D}$ level and sleep apnea finding in all 269 patients.

the association and relation of vitamin $\mathrm{D}$ to different medical and sleep disorders. In fact although there is an anatomic and epidemiological connection between sleep disorders and vitamin D deficiency, makes little sense if vitamin D's actions are thought to be limited to bone and calcium metabolism.

The results in this study showed no obvious relation, other than worsening symptoms or severity of OSAS, thus a patient with OSAS do suffer more if they had Vitamin D deficiency, it is may considered as augmenting effect but not causative effect. Thus furthermore would difficult to treat or tolerate CPAP, may benefit from vitamin D supplement this prompt other clinical studies and trials to answer, as synergistic effect, although may or should not replace or over took CPAP. Vitamin D supplementation was shown to improve insulin release and action in some studies; however, it is controversial. Regarding to these mechanistic links, vitamin D deficiency may have a potential role in pathogenesis and/or metabolic outcomes of OSA in terms of inflammatory response and IR. The therapeutic effects together with the anatomic-functional correspondence warrant further investigation and consideration of vitamin $\mathrm{D}$ in the etiology and therapy of sleep 
Citation: Shendi F, Mahboub B, Alhariri H, Soans A (2016) Prevalence of Vitamin D Deficiency in Obstructive Sleep Apnea Disorder in Dubai, UAE. J Clin Respir Dis Care 2: 112. doi: 10.4172/ 2472-1247.1000112

\begin{tabular}{|c|c|c|c|c|}
\hline OSA Severity (all patients) & Total & \multicolumn{2}{|c}{ Vitamin D problem } \\
& Not done & Vitamin D insufficiency & Vitamin D deficiency \\
\cline { 2 - 4 } & $4(13.3 \%)$ & $16(53.3 \%)$ & $10(33.4 \%)$ \\
\hline No OSA & $6(13.3 \%)$ & $28(62.3 \%)$ & $11(24.4 \%)$ \\
\hline Mild & $4(17.5 \%)$ & $16(69.5 \%)$ & $3(13 \%)$ \\
\hline Sederate & $9(23 \%)$ & $24(61.5 \%)$ & $6(100 \%)$ & $23(100 \%)$ \\
\hline Total & 23 & 84 & $39(100 \%)$ & 30 \\
\hline
\end{tabular}

Table 4: The relationship between vitamin $D$ level and sleep apnea finding in 144 patients who had the vitamin $D$ level checked per sleep apnea severity.

\begin{tabular}{|c|c|c|c|}
\hline OSA Severity (all patients) & Total & \multicolumn{2}{|c|}{ Vitamin D problem } \\
\cline { 2 - 4 } & Not done & Vitamin D insufficiency & Vitamin D deficiency \\
\hline No OSA & $4(17.4 \%)$ & $16(19 \%)$ & $10(33.4 \%)$ \\
\hline Mild & $6(26 \%)$ & $28(33.3 \%)$ & $11(36.6 \%)$ \\
\hline Moderate & $4(17.4 \%)$ & $16(19 \%)$ & $3(10 \%)$ \\
\hline Severe & $9(39.2 \%)$ & $24(28.7 \%)$ & $6(20 \%)$ \\
\hline Total & $23(100 \%)$ & $84(100 \%)$ & 30 \\
\hline
\end{tabular}

Table 5: The relationship between vitamin $\mathrm{D}$ level and sleep apnea finding in 144 patients who had the vitamin $\mathrm{D}$ level checked per the level of vitamin $\mathrm{D}$.

disorders.

Furthermore totality of vitamin D actions are need to be understood, then it may seems sense to that the hormone that links us to the sun would also affect circadian sleep cycle, taking in consideration indirect or overlapping effect of vitamin D on other metabolic syndrome which may associate or cross over with the effect may result from OSAS on those metabolic disorders as Diabetes Mellitus and obesity.

\section{Summary}

When the severity of OSAS increases, vitamin D deficiency or insufficiencybecomes more pronounced. Thus subjects with more severe OSA indices tended to present lower vitamin D levels; furthermore Vitamin D deficiency may play a role and/or worsen OSA's. We need to study more OSA adverse outcomes on glucose metabolism, in relation to vitamin D deficiency \& insufficiency, as may be considered for supplementation treatment which was shown to ameliorate abnormal glucose metabolism and inflammation.

Obstructive sleep apnea (OSA) and vitamin D deficiency are two separate disorders associating with obesity, inflammation, and impaired glucose metabolism. Much remains to be learned concerning the complex relationship between chronically low levels of vitamin $\mathrm{D}$, normal sleep, sleep disruption, and daytime neurocognitive impairment.

\section{References}

1. (2010) Dietary Reference Intakes for Calcium, Phosphorus, Magnesium, Vitamin D, and Fluoride. Standing Committee on the Scientific Evaluation of Dietary Reference Intakes Food and Nutrition Board, Institute of Medicine.

2. Nair S (2010) Symptoms of low vitamin D levels.

3. Stumpf WE, O'Brien LP (1987) $1,25(\mathrm{OH})_{2}$ vitamin D3 sites of action in the brain. An autoradiographic study. Histochemistry 87: 383-406.

4. Kapur VK (2010) Obstructive sleep apnea: diagnosis, epidemiology, and economics. Respir Care. 55: 1155-1167.

5. Mete T, Yalcin Y, Berker D, Ciftci B, Guven SF, et al. (2013) Obstructive sleep apnea syndrome and its association with vitamin $D$ deficiency. J Endocrinol Invest 36: 681-685.

6. Bozkurt NC, Cakal E, Sahin M, Ozkaya EC, Firat H, et al. (2012) The relation of serum 25-hydroxyvitamin-D levels with severity of obstructive sleep apnea and glucose metabolism abnormalities. Endocrine 41: 518-525.

7. McCarty DE, Chesson AL Jr, Jain SK, Marino AA (2013) The link between vitamin D metabolism and sleep medicine. Sleep Med Rev 18: 311-319.

8. Gominak SC, Stumpf WE (2012) The world epidemic of sleep disorders is linked to vitamin D deficiency. Med Hypotheses 79: 132-135.

9. Tasali E, Mokhlesi B, Van Cauter E (2008) Obstructive sleep apnea and type 2 diabetes: interacting epidemics. Chest 133: 496-506.

10. Tasali E, Ip MS (2008) Obstructive sleep apnea and metabolic syndrome: alterations in glucose metabolism and inflammation. Proc Am Thorac Soc 5: 207-217. 